\title{
Estimating Entropy of Liquids from Atom-Atom Radial Distribution Functions: Silica, Beryllium Fluoride and Water
}

\author{
Ruchi Sharma, Manish Agarwal and Charusita Chakravarty* \\ Department of Chemistry, \\ Indian Institute of Technology-Delhi, \\ New Delhi: 110016, India.
}

\begin{abstract}
Molecular dynamics simulations of water, liquid beryllium fluoride and silica melt are used to study the accuracy with which the entropy of ionic and molecular liquids can be estimated from atom-atom radial distribution function data. The pair correlation entropy is demonstrated to be sufficiently accurate that the density-temperature regime of anomalous behaviour as well as the strength of the entropy anomaly can be predicted reliably for both ionic melts as well as different rigid-body pair potentials for water. Errors in the total thermodynamic entropy for ionic melts due to the pair correlation approximation are of the order of $10 \%$ or less for most state points but can be significantly larger in the anomalous regime at very low temperatures. In the case of water, the rigid-body constraints result in larger errors in the pair correlation approximation, between 20 and 30\%, for most state points. Comparison of the excess entropy, $S_{e}$, of ionic melts with the pair correlation entropy, $S_{2}$, shows that the temperature dependence of $S_{e}$ is well described by $T^{-2 / 5}$ scaling across both the normal and anomalous regimes, unlike in the case of $S_{2}$. The residual multiparticle entropy, $\Delta S=S_{e}-S_{2}$, shows a strong negative correlation with tetrahedral order in the anomalous regime.
\end{abstract}

* Author for correspondence (Tel: (+) 91112659 1510; Fax: (+) 91112686 2122; E-mail: charus@chemistry.iitd.ernet.in) 


\section{INTRODUCTION}

The thermodynamic excess entropy of a fluid is defined as the difference in entropy between the fluid and the corresponding ideal gas under identical temperature and density conditions. In the case of a classical fluid, the excess entropy corresponds to the lowering of the entropy of the fluid, relative to the ideal gas, due to the presence of multi-particle positional correlations ${ }^{1}$. The total entropy of a classical fluid can be written as $\underline{2}, \underline{3}, \underline{4}, 5, \underline{6}, \underline{7}, 8,10$

$$
S=S_{i d}+S_{2}+S_{3}+\ldots=S_{i d}+\sum_{n=2}^{\infty} S_{n}
$$

where $S_{i d}$ is the entropy of the ideal gas reference state, $S_{n}$ is the entropy contribution due to $n$-particle spatial correlations and the excess entropy is defined as, $S_{e}=S-S_{i d}$. Such a multiparticle expansion of the entropy is interesting because it allows for the prediction of thermodynamic properties from structural correlation functions. Moreover, semiquantitative excess-entropy based scaling relationships of the form

$$
X=A \exp \left(-\alpha S_{e}\right)
$$

where $X$ is a suitably scaled transport property $11,12,13,14,15$ allow for the possibility of making interesting connections between structural, thermodynamic and transport properties of fluids.

Neutron or X-ray scattering experiments can provide atom-atom radial distribution functions (RDFs), $g_{\alpha \beta}(r)$, associated with the probability of finding an atom of species $\beta$ at a distance $r$ from an atom of species $\alpha$ relative to the probability in the corresponding ideal gas ${ }^{1}$. In terms of the atom-atom radial distribution function, one can write an ensembleinvariant expression for the pair correlation contribution to the entropy of a multicomponent fluid of $N$ particles enclosed in a volume $V$ at temperature $T$ as $\frac{16,17}{6}$

$$
S_{2} / N k_{B}=-2 \pi \rho \sum_{\alpha, \beta} x_{\alpha} x_{\beta} \int_{0}^{\infty}\left\{g_{\alpha \beta}(r) \ln g_{\alpha \beta}(r)-\left[g_{\alpha \beta}(r)-1\right]\right\} r^{2} d r
$$

where $x_{\alpha}$ is the mole fraction of component $\alpha$ in the mixture and $\rho$ is the number density of the fluid. Note that the excess entropy is measured in this case with respect to the entropy of an ideal gas consisting of a non-interacting mixture of spherical particles with entropy $S_{i d}$ given by

$$
S_{i d} / N k_{B}=1+\sum_{\alpha} x_{\alpha}\left[0.5 v_{\alpha}-\ln \left(\rho_{\alpha} \Lambda_{\alpha}^{3}\right)\right]
$$


where $v_{\alpha}$ is the number of degrees of freedom associated with the component $\alpha$ and $\Lambda_{\alpha}$ is the thermal de Broglie wavelength of component $\alpha$. The multiparticle expansion of the entropy has been studied most extensively for simple liquids and liquid mixtures of structureless particles with isotropic interactions, contribution captures 85-90\% of the excess entropy, with the largest discrepancy occurring at intermediate densities ${ }^{8}$.

In the case of a homogeneous, isotropic molecular fluids, an alternative and physically reasonable reference state for the multiparticle expansion is provided by an ideal gas of rigid rotors. In this case, pair correlation contribution to the entropy must be formulated in terms of orientationally-dependent pair correlation functions, $g\left(r, \omega^{2}\right)$, where $\omega^{2}$ denote the relative orientations of the two molecules $\underline{18} \underline{\underline{19}}$. The orientation-dependent pair correlation It is convenient to perform a further decomposition of $g\left(r, \omega^{2}\right)$ as

$$
g\left(r, \omega^{2}\right)=g(r) g\left(\omega^{2} \mid r\right)
$$

where $g\left(\omega^{2} \mid r\right)$ is the conditional probability of observing an orientational separation $\omega^{2}$ at a relative separation $r$ of the molecular centres of mass. The pair correlation entropy of a molecular system, denoted by $S_{2}^{m o l}$, may then be written as a sum of the translational entropy, $S_{2}^{t r}$, dependent only on $g(r)$, and orientational contribution, $S_{2}^{o r}$, involving a suitable weighted average over $g\left(\omega^{2} \mid r\right)$. While simulations show that this procedure yields reasonable estimates of the entropy for standard pair-additive water models, orientation-dependent pair correlation functions are not directly accessible from experiment. Since evaluation of the correlation functions from simulations with adequate statistical accuracy may prove complicated, a number of approximate factorizations of $g\left(r, \omega^{2}\right)$ have been studied $\underline{20}, 21,22$.

Since atom-atom radial distribution functions (RDFs) are obtainable from experiments, obtaining estimates of thermodynamic and transport properties of liquids from atom-atom RDFs is very attractive because it allows one to directly connect structural information with thermodynamic and transport properties of fluids. In principle, such studies could contribute to improving reverse Monte Carlo strategies for structure determination as well as multiscale methodologies for constructing coarse-grained potentials $23,24,25,26,27,28,29$. In order to develop this possibility, it is necessary to test the numerical validity of the pair correlation approximation for a wider range of systems than simple atomic liquids. The purpose of this paper is to examine the extent to which the pair correlation entropy, computed from atomatom RDFs, captures the temperature and density dependence of the excess entropy for ionic melts and molecular liquids. We also study the behaviour of the residual multiparticle 
entropy (RMPE), defined as $\Delta S=S_{e}-S_{2}$, since it has been shown contain physically significant information on local order in a liquid close to phase transitions. For example, the state point for which the RMPE crosses from negative to positive is found to be close to ordering phase transitions, such as freezing and the isotropic-nematic transition $10,14,30,31$.

Ionic melts are of considerable technological importance and are, as a consequence, wellstudied experimentally as well as computationally 32,33 . Many ionic melts form random liquid state networks and show interesting deviations from simple liquid behaviour in their thermodynamic and transport properties $34,35,36,37,38$. Other than a limited comparison in the case of liquid silica ${ }^{39}, \underline{41}$, we are not aware of any systematic testing of the pair correlation approximation for the entropy in the case of ionic melts. As representative examples of ionic melts, we choose beryllium fluoride $\left(\mathrm{BeF}_{2}\right)$ and silica $\left(\mathrm{SiO}_{2}\right)$. Molecular liquids represent an interesting test case for estimating entropy using atom-atom RDF data, since the pair correlation approximation, as defined in equation (3), is expected to be poorer because the bond angle contraints give rise to strong three-body correlations and an ideal gas of rigid molecules is a more appropriate reference state, than a mixture of monoatomic ideal gases as implicit in equation (3). Nonetheless, the experimentally accessible quantities are the atom-atom RDFs and it is interesting to see how much information can be extracted from these quantities. As an example of a molecular liquid, we choose water which is of obvious interest as a pure liquid as well as a solvent, and is representative of a wider class of hydrogen-bonded solvents $2,43,44,45$. Computing the entropy of a molecular fluid from atomatom pair correlation data has not been attempted, though orientationally dependent pair correlation functions, which are not accessible experimentally, have been used to estimate the entropy of several commonly used model water potentials 21,46 .

All three liquids that we study in this work $\left(\mathrm{H}_{2} \mathrm{O}, \mathrm{SiO}_{2}\right.$ and $\left.\mathrm{BeF}_{2}\right)$ have similar anomalous thermodynamic and kinetic properties $34,35,36,37,38,39,40,41,47,48,49,50,51,52,53$. The most obvious signature of the thermodynamic anomalies is the existence of a regime of anomalous density behaviour where the isothermal expansion coefficient, $\alpha=\left(1 / V_{m}\right)\left(\partial V_{m} / \partial T\right)_{P}$ is negative. The region of the density anomaly is bounded by temperatures of maximum and minimum density for which $\alpha=0$. While the temperature of minimum density is experimentally difficult to observe, the locus of temperatures of maximum density (TMD) can be traced in the density-temperature or temperature-pressure plane for many systems. The maximum temperature and maximum density along this locus, $T_{T M D}^{\max }$ and $\rho_{T M D}^{\max }$ respectively, can be thought of as upper bounds above which the thermal kinetic energy or degree of compression 
respectively is sufficient that the liquid conforms to thermodynamic behaviour characteristic of simple liquids. Note that the lowest temperature or maximum density that can be obtained on the TMD locus will be limited by spontaneous crystallisation or glass formation. The diffusional anomaly corresponds to the set of state points for which the diffusivity increases with density. A unified explanation for the thermodynamic and kinetic anomalies can be found based by existence of an excess entropy anomaly involving a rise in excess entropy on isothermal compression $39,40,41,54,55,56$. The relationship between excess and pair correlation entropies in these liquids is of special interest from the point of view of comparing the two quantities in the normal and anomalous regimes, since there is some indication that anomalous colloidal fluids with core-softened interactions show a greater magnitude of the RMPE contribution at high densities ${ }^{56}$. In the case of water, simulation results for TIP4P water suggest that the RMPE is close to zero close to the TMD at 1 atm pressure.

Molecular dynamics simulations for $\mathrm{BeF}_{2}$ and $\mathrm{SiO}_{2}$ are performed using the transferable rigid ion model (TRIM) $)^{51,57,58,59}$ and van Beest-Kramer-van Santen (BKS) $\underline{49,60,61}$ potentials respectively. For both the systems, the ideal gas limit is defined as an $\mathrm{MX}_{2}$ binary mixture of uncharged particles with the same masses as the corresponding ionic melts but with zero interparticle interactions. The excess entropy, $S_{e}$, with respect to this ideal gas is evaluated using thermodynamic integration. Using the radial distribution functions extracted from the simulations, one can obtain both the pair correlation entropy $\left(S_{2}\right)$ and the residual multiparticle entropy (RMPE), given by $\Delta S=S_{e}-S_{2}$. In the case of water, we have simulated three of the commonly used rigid-body, effective pair interaction models $\left(\mathrm{SPC} / \mathrm{E}^{62}\right.$, TIP3P ${ }^{63}$ and TIP5P $\left.{ }^{64}\right)$ and evaluated the entropy from the $g_{O O}(r), g_{H H}(r)$ and $g_{O H}(r)$ radial distribution functions. Table I shows the $T_{T M D}^{\max }$ and $\rho_{T M D}^{\max }$ values for BKS silica, TRIM BeF 2 and the SPC/E and TIP5P water models as determined in previous studies $40,50,53,65,66$.

The paper is organised as follows. Section II summarises the computational details associated with the molecular dynamics simulations of silica, beryllium fluoride and water that we have performed. Section III describes the thermodynamic integration procedure that we have followed to obtain the thermodynamic entropy of the two $\mathrm{MX}_{2}$ ionic melts. In the case of water, thermodynamic entropies for standard potentials are available in the literature. Section IV discusses the results for the two ionic melts while Section V contains the results for water. Conclusions are summarised in Section VI. 


\section{COMPUTATIONAL DETAILS}

For all the three systems, we use parametric effective pair potentials. Each of these interaction models has been well-studied in the literature in terms of behaviour on supercooling, glass transition and waterlike structural, kinetic and thermodynamic anomalies. For convenience, we describe the functional forms of the pair potentials and give the parameters in Tables II, III and IV. The molecular dynamics simulations were performed using the DL_POLY software package $e^{67,68}$.

\section{Model Potential for Silica}

To model the interatomic interactions in silica, we use the van Beest-Kramer-van Santen (BKS) potential with an additional 30-6 Lennard-Jones type correction term $\$ 9,60,61$. The pair interaction between atoms $i$ and $j$ is given by:

$$
\phi_{B K S}\left(r_{i j}\right)=\frac{q_{i} q_{j}}{4 \pi \varepsilon_{0} r_{i j}}+A_{i j} \exp ^{-b_{i j} r_{i j}}-\frac{C_{i j}}{r_{i j}^{6}}+4 \epsilon_{i j}\left[\left(\frac{\sigma_{i j}}{r_{i j}}\right)^{30}-\left(\frac{\sigma_{i j}}{r_{i j}}\right)^{6}\right]
$$

where $r_{i j}$ is the distance between atoms $i$ and $j$ carrying charges $q_{i}$ and $q_{j}, A_{i j}, b_{i j}$ and $C_{i j}$ are the parameters associated with the Buckingham potential for short-range repulsiondispersion interactions and $\epsilon_{i j}$ and $\sigma_{i j}$ are the energy and length scale parameters for the 30-6 Lennard-Jones interaction. The parameters for the modified BKS potential used in this work are given in Table II.

\section{Model Potential for Beryllium fluoride}

We use the transferable rigid ion model (TRIM) potential for interatomic interactions in beryllium fluoride $51,57,58,59$. The pair interaction between atoms $i$ and $j$ is given by:

$$
\phi_{T R I M}\left(r_{i j}\right)=\frac{z_{i} z_{j} e^{2}}{4 \pi \varepsilon_{o} r_{i j}}+\left(1+\frac{z_{i}}{n_{i}}+\frac{z_{j}}{n_{j}}\right) b \exp \left(\frac{\sigma_{i}+\sigma_{j}-r_{i j}}{s}\right)
$$

where $r_{i j}$ is the distance between atoms $i$ and $j$. The parameters associated with an atom of type $l$ are the charge $z_{l}$, the number of valence-shell electrons $n_{l}$ and the ionic size parameter $\sigma_{l}$. The repulsion parameter $b$ and the softness parameter $s$ are assumed to be the same for all three types of pair interactions in $\mathrm{BeF}_{2}$. The parameters for the TRIM potential used in this work are given in Table III. 


\section{Model Potentials for Water}

All rigid-body, effective pair potentials for water assume that the molecule can be represented by a single Lennard-Jones site located on the oxygen atoms and model the charge distribution by a set of distributed charges with a fixed geometry. The parametric form of the interaction between two water molecules $a$ and $b$ is given by:

$$
U_{a b}=\sum_{i} \sum_{j} \frac{q_{i} q_{j}}{r_{i j}}+4 \epsilon\left(\frac{\sigma^{12}}{r_{O O}^{12}}-\frac{\sigma^{6}}{r_{O O}^{6}}\right)
$$

where $i$ and $j$ index partial charges located on molecules $a$ and $b$ respectively and $r_{O O}$ refers to the distance between the oxygen atoms of the two monomers.

The SPC/E and TIP3P models use three charged sites which are located at the atomic positions and are associated with the corresponding atomic masses. The TIP5P model retains the three atomic sites and uses two additional massless sites to represent the oxygen lone pair electron density distribution. The potential parameters for the three water models are summarised in Table IV.

\section{Molecular Dynamics}

Molecular Dynamics simulations for all three systems were carried out in the canonical $(N-V-T)$ ensemble, using the DL_POLY software package $e^{67}, 68$, under cubic periodic boundary conditions. The effects of electrostatic (long-range) interactions were accounted for by the Ewald summation method ${ }^{69,70}$. The non-coulombic part was truncated and shifted at $7.5 \AA$ for $\mathrm{SiO}_{2}$ and $\mathrm{BeF}_{2}$ and at $9.0 \AA$ for $\mathrm{H}_{2} \mathrm{O}$. A Berendsen thermostat, was used to maintain the desired temperature for the production run. The leapfrog Verlet algorithm with a time step of $1 \mathrm{fs}$ was used to integrate the equations of motion; in the case of water, the SHAKE algorithm was used to impose the rigid-body contraints. The MD simulation details, including the lengths of the equilibration and production runs, are summarised in Table V. The cutoff distance for the radial distribution functions was kept the same as the potential cutoff and the bin size was adjusted so that trapezoidal qaudrature to evaluate the pair correlation contribution to the entropy using equation (3) resulted in less than $1 \%$ error. 


\section{ESTIMATION OF THERMODYNAMIC ENTROPY OF IONIC MELTS}

We follow the thermodynamic integration procedure described previously by SaikiaVoivod and co-workers to evaluate the thermodynamic excess entropy of BKS silica where the full Ewald summation was replaced by smoothly tapering off to zero the realspace and short-range contributions to the interaction and removing the reciprocal space contribution $47,48,49,52$. The internal energy differences between the short-range and full Ewald versions of the BKS were of the order of $0.2 \%$ or less. Since we use the full Ewald version of the potential, it was, however, necessary to recompute the thermodynamic entropy of BKS silica. To our knowledge, the entropy of the TRIM model of $\mathrm{BeF}_{2}$ has so far not been evaluated.

The model potentials used here envisage the ionic melts as being fluids composed of particles with long-range Coulomb interactions and short-range repulsions. Such a Coulombic system cannot be reversibly transformed into an ideal gas reference system. Therefore, a binary Lennnard-Jones (BLJ) system with an $\mathrm{MX}_{2}$ stoichiometry was introduced as an intermediate state. The entropy of the ionic melt at a given state point $\left(V_{0}, T_{0}\right)$ was first evaluated relative to the binary Lennard-Jones system at the same state point. The entropy of the BLJ liquid at $\left(V_{0}, T_{0}\right)$ was then evaluated relative to the ideal gas limit at $\left(V_{\infty}, T_{0}\right)$ where $V_{\infty}$ corresponds to such a large volume that the fluid can be regarded as an ideal gas.

To determine the entropy of the ionic melt relative to the BLJ liquid, thermodynamic integration was performed with respect to a Kirkwood-type coupling parameter, $\lambda$, which interpolates between the potential energy function, $U(\mathbf{r})$, of the BLJ liquid and the ionic melt such that 69

$$
U_{\lambda}(\mathbf{r})=(1-\lambda) U_{B L J}(\mathbf{r})+\lambda U_{M X_{2}}(\mathbf{r})
$$

The free energy difference between the BLJ and $M X_{2}$ ionic melt at $\left(V_{0}, T_{0}\right)$ can be written as:

$$
\Delta F\left(V_{0}, T_{0}\right)=\int_{0}^{1}\left\langle\frac{\partial U_{\lambda}}{\partial \lambda}\right\rangle_{\lambda} d \lambda=\int_{0}^{1}\left\langle U_{M X_{2}}-U_{B L J}\right\rangle_{\lambda} d \lambda
$$

The integrand $\langle\cdots\rangle_{\lambda}$ in the above equation refers to a canonical ensemble average for a system described by the potential energy function $U_{\lambda}(\mathbf{r})$. The integral was evaluated using trapezoidal quadrature with the value of the integrand at specific values of the variable $\lambda$ being computed using a canonical ensemble MD simulation with volume $V_{0}$, temperature $T_{0}$ and interaction potential $U_{\lambda}(\mathbf{r})$. The entropy of system $M X_{2}$ at this state point, denoted 
by $S_{M X_{2}}\left(V_{0}, T_{0}\right)$, then be evaluated using,

$$
S_{M X_{2}}\left(V_{0}, T_{0}\right)=S_{B L J}\left(V_{0}, T_{0}\right)+\frac{1}{T}\left[U_{M X_{2}}\left(V_{0}, T_{0}\right)-U_{B L J}\left(V_{0}, T_{0}\right)-\Delta F\left(V_{0}, T_{0}\right)\right]
$$

The entropy of the BLJ system relative to the binary ideal gas is obtained as

$$
S_{B L J}\left(V_{0}, T_{0}\right)=S_{i d}\left(V_{0}, T_{0}\right)+\frac{1}{T}\left[\left\langle U_{B L J}\left(V_{0}, T_{0}\right)\right\rangle-\int_{V_{0}}^{V_{\infty}} P_{L J}^{e x} d V\right]
$$

where $S_{i d}$ is the entropy of the ideal gas as defined in equation (4) The third term in the above expression is obtained by evaluating the excess pressure of the BLJ system for increasing system volumes using simulations at moderate to low densities and a virial expansion at very low densities.

Once the entropy of the ionic melt has been obtained at a given state point $\left(V_{0}, T_{0}\right)$ using the above procedure, the entropy at an arbitrary state point $(V, T)$ can be obtained as

$$
S(V, T)=S\left(V_{0}, T_{0}\right)+\Delta S_{T}+\Delta S_{V}
$$

where $\Delta S_{T}$ is entropy change for isothermal change in volume from $V_{0}$ to $V$ and $\Delta S_{V}$ is the entropy change for isochoric change in temperature from $T_{0}$ to $T$. The following expression can be used to evaluate $\Delta S_{T}$ :

$$
\Delta S_{T}=\frac{1}{T_{0}}\left[U_{M X_{2}}\left(V, T_{0}\right)-U_{M X_{2}}\left(V_{0}, T_{0}\right)+\int_{V_{0}}^{V} P\left(V^{\prime}\right) d V^{\prime}\right]
$$

where $P$ is the pressure of the $\mathrm{MX}_{2}$ liquid. To evaluate $\Delta S_{V}$, we use

$$
\Delta S_{V}=\int_{T_{0}}^{T} \frac{1}{T^{\prime}}\left(\frac{\delta E}{\delta T^{\prime}}\right) d T^{\prime}
$$

where $E$ is the total internal energy which must be the sum of the classical kinetic $\left(1.5 N k_{B} T\right)$ and potential $\left(\left\langle U_{M X_{2}}\right\rangle\right)$ energy contributions for the $N$ particle system. Since the ensemble average of the potential energy for $\mathrm{BKS}$ silica as well as TRIM $\mathrm{BeF}_{2}$ can be fitted by functional form, $a(V)+b(V) T^{3 / 5}$, the integrand in equation (14) is simple to evaluate.

Since the internal energy estimates obtained using the short-range BKS and the BKS potential with explicit Ewald summation used here are very small, we used the same BLJ reference state as used by Saika-Voivod and co-workers. The potential energy parameters for this BLJ system, referred to as $\mathrm{BLJ}_{\mathrm{SiO}_{2}}$, are listed in Table VI. and the entropy of this system at $4000 \mathrm{~K}$ and $2.307 \mathrm{~g} \mathrm{~cm}^{-3}$ was previously evaluated as $84.028 \mathrm{~J} \mathrm{~mol}^{-1} \mathrm{~K}^{-1}$. For this state point, we used equations (13) and (14) in conjunction with molecular dynamics 
simulations of systems at different values of $\lambda$ to compute the entropy of BKS silica as 76.82 $\mathrm{J} \mathrm{K}^{-1}$, which differs by just $2.5 \%$ from the entropy of short-range BKS silica. The entropies of BKS silica over the entire range of temperature and density were then calculated relative to this state point.

Given the stoichiometry and the similar radius ratios of $\mathrm{BeF}_{2}$ and $\mathrm{SiO}_{2}$, it was possible to rescale the $\mathrm{BLJ}_{\mathrm{SiO}_{2}}$ in order to define an appropriate reference system for $\mathrm{BeF}_{2}$, denoted by $\mathrm{BLJ}_{\mathrm{BeF}_{2}}$. Since the locations of the first minima in the Be-Be, F-F and Be-F RDFs are very similar to those in the corresponding RDFs for silica, the length scale parameters for the $\mathrm{BLJ}_{\mathrm{BeF}_{2}}$ state system were kept the same as for $\mathrm{BLJ}_{\mathrm{SiO}_{2}}$. The relative well-depths of the three LJ-type pair interactions were also kept the same. Therefore if $\epsilon_{M-X}$ and $\sigma_{M-X}$ are chosen as the units of energy and length respectively, then the two BLJ systems would have identical internal energies and entropies at the same number density and reduced temperature. To set a suitable energy scale for the BLJ system, we use $\epsilon_{B e-F} \approx \epsilon_{S i-O}\left(T_{T M D, B e F_{2}}^{\max }\right) /\left(T_{T M D, S i O_{2}}^{\max }\right)$ and set $\epsilon_{\mathrm{Be}-\mathrm{F}}$ as $14.21 \mathrm{~K}$, as shown in Table VI. As discussed above, the $\mathrm{BLJ}_{\mathrm{SiO}_{2}}$ liquid at $4000 \mathrm{~K}$ and $2.307 \mathrm{~g} \mathrm{~cm}^{-3}$ has total entropy equal to $84.028 \mathrm{~kJ} \mathrm{~mol}^{-1} \mathrm{~K}^{-1}=10.106 k_{B}$, excess entropy of $9.23 k_{B}$ and internal energy of $56.40 \epsilon_{S i-O}$. The equivalent state point for $\mathrm{BeF}_{2}$ melt would be at $1777 \mathrm{~K}$ and $1.805 \mathrm{~g} \mathrm{~cm}^{-3}$ which would have the same internal energy and excess entropy in reduced units. In order to calculate the total entropy, the ideal gas contribution must be computed using masses of Be and $\mathrm{F}$. The total entropy of BLJ $\mathrm{BeF}_{2}$ was then found to be $70.7 \mathrm{~J} \mathrm{~mol}^{-1} \mathrm{~K}^{-1}=8.5 k_{B}$ at this state point. The entropies over the entire range of temperature and density for $\mathrm{BeF}_{2}$ were then calculated relative to this state point.

\section{IONIC MELTS: SILICA AND BERYLLIUM FLUORIDE}

Figure 1 shows the thermodynamic excess entropy as a function of density for $\mathrm{SiO}_{2}$ and $\mathrm{BeF}_{2}$ melts. Unlike the monotonic decrease in $S_{e}$ with $\rho$ seen in simple liquids, both systems have a well-defined anomalous regime where excess entropy rises with increasing density. The anomalous behaviour is more pronounced for the low temperature isotherms where the anisotropic interactions stabilizing local tetrahedral order are large in comparison to thermal kinetic energies. All the $S_{e}(\rho)$ curves have a well-defined maximum beyond which the excess entropy decreases with density; the maxima are more pronounced for the low temperature isotherms with $T<T_{T M D}^{\max }$ but can be identified in the high temperature isotherms as well.

It is useful to compare the $S_{e}(\rho)$ behaviour with the density dependence of the pair 
correlation entropy, $S_{2}$, for $\mathrm{SiO}_{2}{ }^{39}$ and $\mathrm{BeF}_{2}{ }^{40}$. The maxima in $S_{e}(\rho)$ and $S_{2}(\rho)$ curves are located at essentially the same value of density within simulation error. For example,

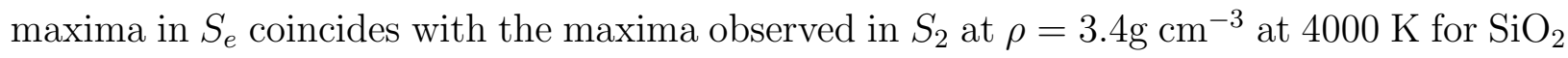
and at $\rho=2.82 \mathrm{~g} \mathrm{~cm}^{-3}$ at $1500 \mathrm{~K}$ for $\mathrm{Be} F_{2}$. The most notable qualitative difference between the $S_{e}(\rho)$ and $S_{2}(\rho)$ curves is the absence of a minimum in the $S_{e}$ curves at low densities. The $S_{2}(\rho)$ curves for all waterlike systems show a minimum at low densities which coincides with the maximum in the tetrahedral order parameter along the isotherms, indicating that $S_{2}$ is more sensitive to tetrahedral order than $S_{e}$.

Figure 2 shows the temperature dependence of the excess entropy of $\mathrm{BeF}_{2}$ and $\mathrm{SiO}_{2}$ along different isochores. We plot $S_{e}$ as a function of $T^{-2 / 5}$ since earlier density functional as well as simulation studies suggest that the excess entropy of simple liquids, even within the pair correlation approximation, obeys a $T^{-2 / 5}$ scaling 71,72 . Figures $2(\mathrm{a})$ and (b) show that both $\mathrm{SiO}_{2}$ and $\mathrm{BeF}_{2}$ melts show an approximate $T^{(-2 / 5)}$ scaling, with small deviations from the scaling behaviour in the anomalous regime. Note that a $T^{3 / 5}$ scaling predicted for the configurational potential energy is obeyed by both the melts and has been used when performing thermodynamic integration ${ }^{34}$. Interestingly, the pair correlation entropy, $S_{2}$, plotted as a function of $T^{-2 / 5}$, of liquids with waterlike anomalies shows a significant change between on going from the normal to the structurally anomalous regime.

To summarise, the qualitative differences that emerge in the density and temperature dependence of $S_{e}$ and $S_{2}$ are: (i) absence of a minimum in $S_{e}$, unlike the minimum in $S_{2}$ which correlates with the maximum in tetrahedral order and (ii) the temperature dependence of $S_{e}$ along isochores is well described by $T^{-2 / 5}$ scaling across both the normal and anomalous regimes, unlike in the case of $S_{2}$. This suggests that it is important to examine the relationship between the residual multiparticle entropy, $\Delta S$, and tetrahedral order parameter, $q_{\text {tet }}$, as is done below.

Figure 3(a) displays the correlation between pair correlation entropy $S_{2}$ and thermodynamic excess entropy, $S_{e}$, in the case of of $\mathrm{SiO}_{2}$. A strong correlation between $S_{2}$ and $S_{e}$ is seen for the low isotherms of $\mathrm{T}=5000,4500$ and $4000 \mathrm{~K}$ i.e. for $T / T_{T M D}^{\max } \leq 1$. For temperatures greater than $T_{T M D}^{\max }(\mathrm{T}=5500$ and $6000 \mathrm{~K})$, the $S_{2}$ versus $S_{e}$ curve shows two branches, a high density and a low density one. The density demarcating the two branches corresponds to $3.0 \mathrm{~g} \mathrm{~cm}^{-3}$. Figure $3(\mathrm{~b})$ shows the $S_{2}$ versus $S_{e}$ correlation plot for $\mathrm{BeF}_{2}$. Isotherms above $2000 \mathrm{~K}\left(T_{T M D}^{\max }=2310 \mathrm{~K}\right)$, the curves have distinct high and low density branches with the demarcation density being $2.4 \mathrm{~g} \mathrm{~cm}^{-3}$. 
In order to understand the relationship between $S_{e}$ and $S_{2}$, we plot $\Delta S$ as a function of $\rho$ in Figure 4. Several interesting features of the RMPE as a function of density are common to both $\mathrm{BeF}_{2}$ and $\mathrm{SiO}_{2}$ melts. In both systems, $\Delta S$ is essentially constant after a characteristic density $\rho_{T M D}^{\max }$ which marks the maximum density for which the thermodynamically anomalous behaviour in the density is observed in the liquid phase. This corresponds to a density of $3 \mathrm{~g} \mathrm{~cm}^{-3}$ for $\mathrm{SiO}_{2}$ and $2.4 \mathrm{~g} \mathrm{~cm}^{-3}$ for $\mathrm{BeF}_{2}$. In both systems isotherms which lie at or below $T_{T M D}^{\max }$ show a decrease in $\Delta S$ with increasing $\rho$ till $\rho_{T M D}^{\max }$ is reached. In contrast, isotherms which lie above $T_{T M D}^{\max }$ show an increase in $\Delta S$ till $\rho_{T M D}^{\max }$ is reached.

Figure 4 also shows that the RMPE is negative for all state points in the case of $\mathrm{SiO}_{2}$ but not in the case of $\mathrm{BeF}_{2}$. This may in part be due to the fact that the BKS interaction potential in the case of $\mathrm{SiO}_{2}$ does not include any explicit cation-cation (or $\mathrm{Si}-\mathrm{Si}$ ) interactions. We find that the RMPE contributes about $10 \%$ or less to the excess entropy, $S_{e}$, of the ionic liquids in the normal regime. In the anomalous regime, specially at low temperatures, the contribution of the $\Delta S$ terms can be as large as $30 \%$ of the total excess entropy in terms of the absolute magnitudes.

To assess the quantitative reliability of the pair correlation approximation to obtain the total thermodynamic entropy, Figure 5 shows the quantity $|\Delta S| / S \%$ where $S=S_{i d}+S_{e}$ is the total thermodynamic entropy. The error caused by the pair correlation approximation is of the order of $6 \%$ or less for both ionic melts in the normal regime, but as expected from the behaviour of the RMPE, the error can be greater in the anomalous regime. Other than the $1500 \mathrm{~K}$ isotherm of $\mathrm{BeF}_{2}$, the magnitude of the error for all state points studied is less than $10 \%$.

The above results suggest that the nature of the relationship between the residual multiparticle entropy and structural order is significantly different in the normal and anomalous regime. Therefore, we plot $\Delta S$ as a function of the tetrahedral order parameter $q_{t e t}$ in Figure 6. The local tetrahedral order parameter, $q_{t e t}$, associated with an atom $i$ is defined as

$$
q_{t e t}=1-\frac{3}{8} \sum_{j=1}^{3} \sum_{k=j+1}^{4}\left(\cos \psi_{j k}+1 / 3\right)^{2}
$$

where $\psi_{j k}$ is the angle between the bond vectors $\mathbf{r}_{i j}$ and $\mathbf{r}_{i k}$ where $j$ and $k$ label the four nearest neighbour atoms of the same type $\mathrm{f}^{\underline{\underline{3}}}$. Figure 6 shows that $\Delta S$ has a strong negative correlation with tetrahedral order in this regime. Clearly, the dominant contribution to the RMPE in the anomalous regime is from the three-body terms which are strongly anticorrelated with tetrahedral order. 
We now reconsider the behaviour of $S_{2}$ versus $S_{e}$ at temperatures less than or equal to $T_{T M D}^{\max }$ in Figure 3. $S_{2}$ and $S_{e}$ are strongly correlated and increase with increasing density till $\rho_{\max }$ is reached. The anomalous regime also shows a strong correlation between tetrahedral order and $S_{2}$ or any related measure of structure in the pair correlation function ${ }^{53}$. The effect of the local tetrahedral ordering, imposed in the case of ionic melts from the steric factors associated with the relative ionic radii, is to strongly couple the two and three-body contributions to the excess entropy. For isotherms lying above $T_{T M D}^{\max }$, at low densities $S_{2}$ is essentially constant while $S_{e}$ increases. The multiparticle correlations in this case serve to increase the entropy with increasing density and are essentially uncorrelated with the tetrahedral order. For densities above $\rho_{\max }$ and temperatures above $T_{T M D}^{\max }$, the system behaves essentially as a simple liquid, with $S_{2}$ and $S_{e}$ both showing a negative correlation with density though with a relatively small variation in either entropy or local order.

\section{MODEL POTENTIALS FOR WATER}

We first consider the the pair correlation estimate of the entropy, based on the $g_{O O}(r)$, $g_{O H}(r)$ and $g_{H H}$ pair correlation functions, with the total entropy as estimated from thermodynamic integration for the SPC/E model for water. The experimentally obtained $g_{O H}(r)$ and $g_{H H}(r)$ RDFs will show a sharp, narrow peak corresponding to the intramolecular distances between these atoms. In the case of rigid body molecular dynamics, this peak will reduce to a $\delta$-function. The $S_{2}$ contribution can be computed from equation (3) from the $g(r)$ functions without this additional $\delta$-function contribution.

Figure 7(a) shows the density dependence of the thermodynamic entropy, $S^{*}$, taken from ref. $\frac{52}{2}$, for several isotherms of SPC/E water lying between $210 \mathrm{~K}$ and $300 \mathrm{~K}$. Comparison with the pair correlation estimate, $S^{*} \approx S_{i d}+S_{2}$, shown in Figure $7(\mathrm{~b})$, indicates that the pair correlation entropy shows the correct qualitative behaviour. The anomalous rise in entropy between 0.95 and $1.10 \mathrm{~g} \mathrm{~cm}^{-3}$ is seen in both Figures $7(\mathrm{a})$ and $7(\mathrm{~b})$ for isotherms at 210,220 and $230 \mathrm{~K}$. The quantitative errors introduced by the pair correlation approximation for SPC/E water are assessed by plotting $|\Delta S| / S \%$ where $S$ is the thermodynamic excess entropy taken from ref. $\frac{52}{2}$ as a function of density in Figure $7(\mathrm{c})$. The errors due to the pair correlation approximation at temperatures of $240 \mathrm{~K}$ and above lie between 20 and $30 \%$ and are almost independent of density. Not surprisingly, this is larger than errors observed for the ionic melts in the normal liquid regime. At low temperatures, specially in the 
anomalous regime, there is a strong density dependence of the errors; for example, for the $210 \mathrm{~K}$ isotherm, the error varies between 10 and $60 \%$.

Since the pair correlation entropy provides a very reasonable estimate of the temperaturedensity regime in which thermodynamic anomalies may be expected, we consider the two other effective pair potential models of water known to have very different regimes of anomalous behaviour (Table I). The TIP5P model reproduces the experimental data closely in this respect and shows a $T_{T M D}^{\max }$ value of $282 \mathrm{~K}$. In the case of the SPC/E model, however, the anomalous regime is shifted to temperatures approximately 30 to $40 \mathrm{~K}$ below those see experimentally. In the case of TIP3P, the anomalies appear to occur at even lower temperatures though a detailed mapping of waterlike anomalies has not been performed for this model. The TIP3P model is, however, of considerable interest since it is frequently used to model the aqueous solvent in biomolecular simulations ${ }^{63}$. To illustrate the quantitative differences between the models, one may note that a recent study indicates that the TMD at $1 \mathrm{~atm}$ pressure occurs at 241K, $182 \mathrm{~K}$ and $285 \mathrm{~K}$ for the SPC/E, TIP3P and TIP5P models respectively ${ }^{73}$. Figure 8 shows the density dependence of the pair corrrelation entropy, $S_{2}$, as a function of density for different isotherms for all three models. It is immediately apparent that the thermodynamically anomalous regime can be correctly identified for all three models from this pair correlation information. An excess entropy anomaly exists in the TIP3P, SPC/E and TIP5P models below 200K, 260K and 360K respectively. The strength

of the excess entropy anomaly, as identified by $\left(\partial S_{2} / \partial(\ln \rho)\right)_{T} \underline{\underline{56}}$, is maximal in the TIP $5 \mathrm{P}$ model and least in the TIP3P model.

\section{CONCLUSIONS}

This paper compares the pair correlation entropy $\left(S_{2}\right)$, determined from the atom-atom radial distribution functions with the excess entropy of two ionic melts (liquid silica and beryllium fluoride) and a molecular liquid (water). The three liquids that we have chosen to study show distinct normal and anomalous regimes. The anomalous regime is characterised by significant departures from simple liquid behaviour and shows a rise in thermodynamic entropy on isothermal compression. This anomalous entropy behaviour can be connected to the existence of waterlike structural, kinetic and thermodynamic entropies. The pair correlation entropy is sufficiently accurate that the density-temperature regime of anomalous behaviour as well as the strength of the entropy anomaly can be predicted reliably for both 
ionic melts as well as different rigid-body pair potentials for water. This simple connection between atom-atom radial distribution functions and thermodynamic anomalies predicted by different water models has not been discussed previously in the literature.

To assess the quantitative contribution of the pair correlations to the thermodynamic entropy, we compare the RDF-based $S_{2}$ estimator for ionic melts with the thermodynamic excess entropy, $S_{e}$, measured with respect to an ideal gas mixture. Errors in the total thermodynamic entropy for ionic melts due to the pair correlation approximation are of the order of $6 \%$ in the normal regime but can be significantly larger in the anomalous regime. In the case of water, we compared the total thermodynamic entropy with the estimate based on the atom-atom RDFs. As expected given the rigid-body constraints for molecular liquids, the pair correlation approximation then causes significantly larger errors, between 20 and $30 \%$, in the normal liquid regime.

In the case of ionic melts, the high density limit of the anomalous regime is marked by the maximum in the $S_{e}(\rho)$ curves at a given temperature and this is well reproduced by the $S_{2}(\rho)$ curves. The excess entropy curve, $S_{e}(\rho)$, does not show a minimum, unlike the $S_{2}(\rho)$ curves for which the minimum correlates with the maximum in tetrahedral order. Along an isochore, the temperature dependence of $S_{e}$ is well described by $T^{-2 / 5}$ scaling across both the normal and anomalous regimes. In contrast, $S_{2}$ shows $T^{-2 / 5}$ scaling behaviour only in the normal liquid regime.

Comparison of pair correlation approximation to the excess entropy from scattering data with calorimetric estimates of the entropy can provide interesting insights into the role of multiparticle interactions in different liquids. For example, in the case of ionic melts, we show that the relationship between $S_{2}$ and $S_{e}$ is significantly different in the normal and anomalous regime. Strong, local anisotropic interactions are dominant in the anomalous regime and serve to couple the two- and three-body contributions to the entropy. Thus the correlation between local order metrics and RMPE in simple and anomalous liquids is qualitatively different.

It is useful at this point to compare our results for excess entropy of the three tetrahedral network-forming liquids with the earlier results for $\mathrm{H}_{2} \mathrm{O}$ using an ideal gas of rigid rotors as a reference state $\underline{20}, \underline{31}, \underline{6}$. Clearly the separation of time scales between intramolecular and intermolecular interactions of water justifies the rigid-body ideal gas limit as the physically more reasonable choice. In the case of ionic melts, the molecular limit is not an obvious choice and defining the reference state as a non-interacting mixture of atoms is a reasonable 
option. The greater accuracy of the pair correlation estimator for the excess entropy of ionic melts, as compared to water, is a consequence of the appropriateness of choice of reference state. Both approaches indicate that the contribution of multiparticle interactions to the excess entropy changes qualitatively on going from the normal to the anomalous regime. The translational entropy, as defined in refs. ${ }^{20}, \underline{46}$, corresponds to the contribution of the O-O pair correlation function to the pair correlation entropy as defined in our work. The orientational entropy must correspond to a subset of the three-body terms in our formulation which is supported by the strong correlation of the RMPE with the tetrahedral order parameter.

In conclusion, the results presented in this paper suggest that atom-atom radial distribution functions can be used to construct a reliable, semiquantitative structural estimator for the entropy. The comparison of such a structural estimator for the entropy with calorimetric data could lead to interesting insights into the role of pair and higher-order particle correlations in determining thermodynamic and transport properties of liquids and serve as additional inputs for improving structure prediction by reverse Monte Carlo or related techniques based on RDF data. We also note that there has been considerable interest in developing coarse-graining strategies based on effective pair potentials for complex liquids. For example, isotropic models of water which reproduce the $g_{O O}(r)$ function have been constructed but do have some problems with representability and transferability $\underline{25}, \underline{26}, \underline{27}, \underline{28}, \underline{29}$. It may be interesting to consider coarse graining strategies which modify the RDF data to reproduce the excess entropy of the liquid.

Acknowledgements This work was financially supported by the Department of Science and Technology, New Delhi. Computational support from the Computer Services Centre are gratefully acknowledged. MA and RS thank Indian Institute of Technology Delhi and Council for Scientific and Industrial Research respectively for financial support. The authors would like to thank Ivan Saika-Voivod for clarifications with regard to their published work.

1 J.-P. Hansen and I. R. McDonald, Theory of Simple Liquids (Academic Press, 1986).

2 H. S. Green, The Molecular Theory of Fluids (North-Holland, Amsterdam, 1952).

3 R. E. Nettleton and H. S. Green, J. Chem. Phys. 29, 1365 (1958).

4 H. J. Raveche, J. Chem. Phys. 55, 2242 (1971).

5 D. C. Wallace, J. Chem. Phys. 87, 2282 (1987).

6 A. Baranyai and D. J. Evans, Phys. Rev. A 40, 3817 (1989). 
7 A. Baranyai and D. J. Evans, Phys. Rev. A 42, 849 (1990).

8 I. Borzsak and A. Baranyai, Chem. Phys. 165, 227 (1992).

9 P. V. Giaquinta and G. Giunta, Physica A 187, 145 (1992).

10 P. V. Giaquinta, G. Giunta and S. P. Giarritta, Phys. Rev. A 45, R6966 (1992).

11 Y. Rosenfeld, Phys. Rev. A 15, 2545 (1977).; Chem. Phys. Lett. 48, 467 (1977).

12 Y. Rosenfeld, J. Phys. Cond. Matt. 11, 5415 (1999).

13 M. Dzugutov, Nature 381, 137 (1996).

14 Y. Rosenfeld, Phys. Rev. E. 62, 7524 (2000).

15 J. J. Hoyt, M. Asta and B. Sadigh, Phys. Rev. Lett. 85, 594 (2000).

16 J. A. Hernando, Mol. Phys. 69, 319 (1990).

17 B. B. Laird and A. D. J. Haymet J. Chem. Phys. 97, 2153 (1992); Phys. Rev. A 45, 5680 (1992).

18 T. Lazardis and M. E. Paulaitis, J. Phys. Chem 96, 3847 (1992).

19 T. Lazaridis and M. E. Paulaitis, J. Phys. Chem 98, 635 (1994).

20 T. Lazaridis and M. Karplus, J. Chem. Phys. 105, 4294 (1996).

21 J. Zielkiewicz, J. Chem. Phys. 123, 104501 (2005).; J. Chem. Phys. 124, 109901 (2006).

22 J. Zielkiewicz, J. Phys. Chem. B 112, 7810 (2008).

23 S. J. L. Billinge and I. Levin, Science 316, 56 (2007).

24 D. A. Keen and R. L. McGreevy, Nature 344, 423 (1990).

25 A. A. Louis, J. Phys. Cond. Matt. 14, 9187 (2002).

26 M. E. Johnson, T. Head-Gordon and A. A. Louis J. Chem. Phys. 126, 144509 (2007).

27 T. Head-Gordon and F. H. Stillinger, J. Chem. Phys. 98, 3313 (1993).

28 R. M. Lynden-Bell and T. Head-Gordon, Mol. Phys. 104, 3593 (2006).

29 S. J. Marrink, H. J. Risselada, S. Yefimov, D. P. Tieleman and A. H. de Vries, J. Phys. Chem. B 111, 7812 (2007).

30 D. Costa, F. Saija and P.V. Giaquinta, J. Phys. Chem. B 107, 9514 (2003).

31 F. Saija, A. M. Saitta and P.V. Giaquinta, J. Chem. Phys. 119, 3587 (2003).

32 P. A. Madden and M. Wilson, J. Phys. Cond. Matt. 12, A95 (2000).

33 P. F. McMillan, J. Mater. Chem. 14, 1506 (2004).

34 P. H. Poole, M. Hemmati, and C. A. Angell Phys. Rev. Lett. 79, 2281 (1997).

35 O. Mishima and H. E. Stanley, Nature 396, 329 (1998). 
36 C. A. Angell, R. D. Bressel, M. Hemmati, E. J. Sare and J. C. Tucker, Phys. Chem. Chem. Phys. 2, 1559 (2000).

37 H. Tanaka, Phys. Rev. B 66, 064202 (2002).

38 A. Takada, P. Richet, C. R. A. Catlow and G. D. Price, J. Non-Cryst. Solids 353, 1892 (2007).

39 R. Sharma, S. N. Chakraborty and C. Chakravarty J. Chem. Phys. 125, 204501 (2006).

40 M. Agarwal, R. Sharma and C. Chakravarty J. Chem. Phys. 127, 164502 (2007).

41 M. Agarwal and C. Chakravarty, J. Phys. Chem. B 111, 13294 (2007).

42 A. K. Soper, F. Bruni and M. A. Ricci, J. Chem. Phys. 106, 247 (1997).,

43 A. K. Soper and M. A. Ricci, Phys. Rev. Lett. 84, 2881 (2000).

44 A. K. Soper, Physica B 276, 12 (2000)..

45 A. K. Soper, L. Dougan, J. Crain, and J. L. Finney, J. Phys. Chem. B 110, 3472 (2006).

46 R. Esposito, F. Saija, A. M. Saitta and P. V. Giaquinta Phys. Rev. E. 73, 040502(R) (2006).

47 I. Saika-Voivod, P. H. Poole and F. Sciortino, Nature 412, 514 (2001).

48 I. Saika-Voivod, F. Sciortino and P. H. Poole, Phys. Rev. E. 69, 041503 (2004).

49 I. Saika-Voivod, F. Sciortino and P. H. Poole, Phys. Rev. E. 63, 011202 (2000).

50 M. S. Shell, P. G. Debenedetti and A. Z. Panagiotopoulos, Phys. Rev. E. 66, 011202 (2002).

51 M. Hemmati, C. T. Moyinihan and C. A. Angell, J. Chem. Phys. 115, 6663 (2001).

52 A. Scala, F. W. Starr, E. La Nave, F. Sciortino and H. E. Stanley, Nature 406, 166 (2000).

53 J. R. Errington and P. G. Debenedetti, Nature 409, 318 (2001).

54 J. Mittal, J. R. Errington and T. M. Truskett, J. Chem. Phys. 125, 076102 (2006).

55 J. Mittal, J. R. Errington and T. M. Truskett, J. Phys. Chem. B 110, 18147 (2006).

56 J. R. Errington, T. M. Truskett and J. Mittal, J. Chem. Phys. 125, 244502 (2006).

57 L. V. Woodcock, C. A. Angell, and P. Cheeseman, J. Chem. Phys. 65, 1565 (1976).

58 W. R. Busing, J. Chem. Phys. 57, 3008 (1972).

59 M. Hemmati and C. A. Angell, J. Non-Cryst. Solids 217, 236 (1997).

60 B. W. H. van Beest, G. J. Kramer and R. A. van Santen, Phys. Rev. Lett. 64, 1955 (1990).

61 G. J. Kramer, N. P. Farragher and B. W. H van Beest Phys. Rev. B 43, 5068 (1991).

62 H. J. C. Berendsen, J. R. Grigera, and T. P. Straatsma, J. Phys. Chem 91, 6269 (1987).

63 W. L. Jorgensen, J. Chandrasekhar, J. D. Madura, R. W. Impey and M. L. Klein, J. Chem. Phys. 79, 926 (1983).

64 M. W. Mahoney and W. L. Jorgensen, J. Chem. Phys. 112, 8910 (2000). 
H. E. Stanley, S. V. Buldyrev, N. Giovambattista, E. La Nave, S. Mossa, A. Scala, F. Sciortino, F. W. Starr and M. Yamada, J. Stat. Phys. 110, 1039 (2003).

66 M. Yamada, S. Mossa, H. E. Stanley and F. Sciortino, Phys. Rev. Lett. 88, 195701 (2002).

67 W. Smith and T. R. Forester, J. Mol. Graphics 14, 136, (1996).

68 W. Smith, C. W. Yong and P. M. Rodger, Mol. Simulat. 28, 385 (2002).; The DL_POLY website is http://www.cse.clrc.ac.uk/msi/software/DL_POLY/.

69 D. Frenkel and B. Smit, Understanding Molecular Simulation: From Algorithms to Applications; Academic Press: London, 2002.

70 M. D. Allen and D. J. Tildesley, Computer Simulation of Liquids (Clarendon Press, Oxford, 1986).

71 Y. Rosenfield and P. Tarazona, Mol. Phys. 95, 141 (1998).

72 S. N. Chakraborty and C. Chakravarty, Phys. Rev. E. 76, 011201 (2007).

73 C. Vega and J. L. F. Abascal, J. Chem. Phys. 123, 144504 (2005). 
TABLE I: Maximum temperature and corresponding density for locus of state points corresponding to temperatures of maximum density (TMD) for different isobars. These temperatures and densities mark the maximum in the density-temperature $(\rho-T)$-plane of the curve connecting state points with $(\partial V / \partial T)_{P}=0$. The data for $T_{T M D}^{\max }$ and $\rho_{T M D}^{\max }$ were taken from the literature and the apprpriate reference is cited in the column heading.

\begin{tabular}{ccccc}
\hline & $\mathrm{SPC} / \mathrm{E}^{53}$ & $\mathrm{TIP} 5 \mathrm{P}^{65}, 66$ & $\mathrm{BeF}_{2} \underline{40}$ & $\mathrm{SiO}_{2}{ }^{50}$ \\
\hline$T_{T M D}^{\max } / \mathrm{K}$ & 249 & 282 & 2310 & 4940 \\
$\rho_{T M D}^{\max } / \mathrm{g} \mathrm{cm}^{-3}$ & 0.97 & 1.0 & 1.805 & 2.307 \\
\hline
\end{tabular}

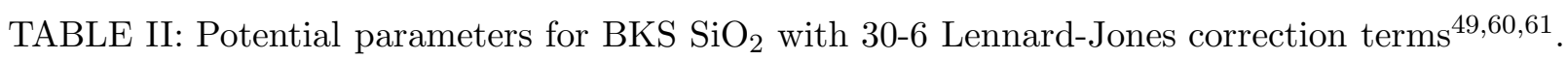

\begin{tabular}{cccccc}
\hline \hline & & & & & \\
$i-j$ & $A_{i j}$ & $b_{i j}$ & $C_{i j}$ & $\epsilon_{i j}$ & $\sigma_{i j}$ \\
& $\left(\mathrm{~kJ} \mathrm{~mol}^{-1}\right)$ & $\left(\AA^{-1}\right)$ & $\left(\mathrm{kJ} \mathrm{mol}^{-1}\right)$ & $\left(\mathrm{kJ} \mathrm{mol}^{-1}\right)$ & $(\AA)$ \\
& & & & & \\
\hline & & & & & \\
S-O & 134015 & 2.76 & 16887.3 & 0.101425 & 1.7792 \\
& & & & & \\
\hline \hline
\end{tabular}

TABLE III: TRIM potential parameters for $\mathrm{BeF}_{2} \underline{51,57,59}$

\begin{tabular}{rrrrr}
\hline \hline$\sigma^{+}$ & $\sigma^{-}$ & $\rho$ & $z^{+} z^{-} n^{+} n^{-}$ & $b$ \\
$(\AA)$ & $(\AA)$ & $\left(\AA^{-1}\right)$ & $\left(\mathrm{kJ} \mathrm{mol}^{-1}\right)$ \\
\hline
\end{tabular}

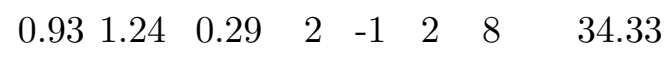


TABLE IV: Comparison of parameters of common water models ${ }^{62,63,64}$. SPC/E and TIP3P are three site models with the respective charges and masses centered at the $\mathrm{O}$ and $\mathrm{H}$ sites $\underline{62}, 63$. TIP5P is a five site model with two additional massless sites $\mathrm{M}^{64}$.

\begin{tabular}{llll}
\hline & SPC $/ \mathrm{E}$ & TIP3P & TIP5P \\
\hline$\epsilon\left(\mathrm{kcal} \mathrm{mol}^{-1}\right)$ & 0.155 & 0.152 & 0.160 \\
$\sigma(\AA)$ & 3.166 & 3.150 & 3.120 \\
$r_{O H}$ & 1.000 & 0.9572 & 0.9572 \\
$\angle H O H(\mathrm{deg})$ & 109.47 & 104.52 & 104.52 \\
$q_{O}$ & -0.8472 & -0.834 & 0.0 \\
$q_{H}$ & 0.4238 & 0.417 & 0.241 \\
$q_{M}$ & - & - & -0.241 \\
$r_{O M}(\AA)$ & - & - & 0.70 \\
$\angle M O M(\mathrm{deg})$ & - & - & 109.47 \\
\hline
\end{tabular}


TABLE V: Computational details of molecular dynamics simulations of $\mathrm{SiO}_{2}, \mathrm{BeF}_{2}$ and $\mathrm{H}_{2} \mathrm{O}$. The simulation cell size is given in terms of the number of formula units, $M$, present in the system. An MD time step of 1 fs was used for all three systems. The time constant for the Berendsen thermostat is denoted by $\tau_{B}$. Equilibriation and production run lengths are denoted by $t_{e q}$ and $t_{\text {prod }}$ respectively.

\begin{tabular}{lccccll}
\hline \hline System & Model & $\mathrm{M}$ & $\begin{array}{c}t_{e q} \\
(\mathrm{~ns})\end{array}$ & $\begin{array}{c}t_{\text {prod }} \\
(\mathrm{ns})\end{array}$ & $\begin{array}{c}\tau_{B} \\
(\mathrm{ps})\end{array}$ & MD algorithm \\
\hline $\mathrm{SiO}_{2}$ & BKS & 150 & $3-6$ & $5-10$ & 200 & Verlet \\
$\mathrm{BeF}_{2}$ & TRIM & 150 & $4-8$ & $6-8$ & 200 & Verlet \\
$\mathrm{H}_{2} \mathrm{O}$ & SPC/E & 256 & 1 & 1 & 10 & Verlet + Quaternion \\
$\mathrm{H}_{2} \mathrm{O}$ & TIP3P & 256 & $0.25-0.5$ & $0.5-0.75$ & 1 & Verlet + SHAKE \\
$\mathrm{H}_{2} \mathrm{O}$ & TIP5P & 256 & $0.25-0.5$ & $0.5-0.75$ & 1 & Verlet + Quaternion \\
\hline
\end{tabular}

TABLE VI: Potential energy parameters for the binary Lennard-Jones fluid used as reference state for thermodynamic integration to obtain entropies of $\mathrm{BKS} \mathrm{SiO}_{2}$ and TRIM $\mathrm{BeF}_{2}$.

\begin{tabular}{cccccc}
\hline & $\mathrm{SiO}_{2}$ & \multicolumn{4}{c}{$\mathrm{BeF}_{2}$} \\
\hline$i-j$ & $\epsilon$ & $\sigma \mathrm{d}$ & $i-j$ & $\epsilon$ & $\sigma$ \\
& $\left(\mathrm{kJ} \mathrm{mol}^{-1}\right)$ & $(\AA)$ & & $\left(\mathrm{kJ} \mathrm{mol}^{-1}\right)$ & $(\AA)$ \\
\hline $\mathrm{Si}-\mathrm{O}$ & 32.0 & 1.6 & $\mathrm{Be}-\mathrm{F}$ & 14.21 & 1.6 \\
$\mathrm{Si}-\mathrm{Si}$ & 23.0 & 3.3 & $\mathrm{Be}-\mathrm{Be}$ & 10.22 & 3.3 \\
$\mathrm{O}-\mathrm{O}$ & 23.0 & 2.8 & F-F & 10.22 & 2.8 \\
\hline
\end{tabular}

Figure Captions

1. Thermodynamic excess entropy, $S_{e}$, as a function of density for (a) $\mathrm{SiO}_{2}$ and (b) $\mathrm{BeF}_{2}$. The vertical line shows the position of minima in the $S_{2}(\rho)$ curves for $\mathrm{SiO}_{2} \underline{39}$ and $\mathrm{BeF}_{2}{ }^{40}$. Unless otherwise stated, entropy is reported in units of $k_{B}$ per atom in all the figures. Isotherms are labelled in degrees $\mathrm{K}$ in all the figures.

2. Temperature dependence of the thermodynamic excess entropy, $S_{e}$, for (a) $\mathrm{SiO}_{2}$ and (b) $\mathrm{BeF}_{2}$ along different isochores. The isochores are labelled by the density in $\mathrm{g} \mathrm{cm}^{-3}$. 
3. Correlation between the pair correlation entopy, $S_{2}$, and the thermodynamic excess entropy, $S_{e}$, for (a) $\mathrm{SiO}_{2}$ and (b) $\mathrm{BeF}_{2}$. The vertical arrows indicate the lowest density for each isotherm studied in this work.

4. Variations in residual multiparticle entropy, $\Delta S$, with density, $\rho$, along different isotherms, labelled by temperature in Kelvin, for (a) $\mathrm{SiO}_{2}$ and (b) $\mathrm{BeF}_{2} . \Delta S$ is reported in units of $k_{B}$ per atom.

5. Percentage contribution of residual multiparticle entropy to the total thermodynamic entropy, $|\Delta S| / S \%$, along different isotherms for (a) $\mathrm{SiO}_{2}$ and (b) $\mathrm{BeF}_{2}$.

6. Correlation between the tetrahedral order parameter, $q$ and the residual multiparticle entropy, $\Delta S$, for (a) $\mathrm{SiO}_{2}$ and (b) $\mathrm{BeF}_{2} . \Delta S$ is reported in units of $k_{B}$ atom. The arrows indicate the lowest density state point along an isotherm simulated in this study.

7. Total entropy of SPC/E water as a function of density. (a) Total thermodynamic entropy, $S$ taken from ref. $\frac{52}{2}$ and (b) total pair correlation entropy, $S^{*}=S_{I d}+S_{2}$ calculated by considering water as a binary mixture of $\mathrm{H}$ and $\mathrm{O}$ atoms. From top to bottom, the isotherms correspond to $\mathrm{T}=300(\nabla), 260(\boldsymbol{\Delta}), 240(\triangle), 230(\bullet), 220(\bigcirc)$ and $210 \mathrm{~K}(\boldsymbol{\square})$. Entropies are reported in units of $k_{B}$ per atom.

8. Pair correlation entropy as a function of density for different model potentials of water: (a) SPC/E (b) TIP3P and (c) TIP5P. Entropies are reported in units of $k_{B}$ per atom. 
FIG. 1:
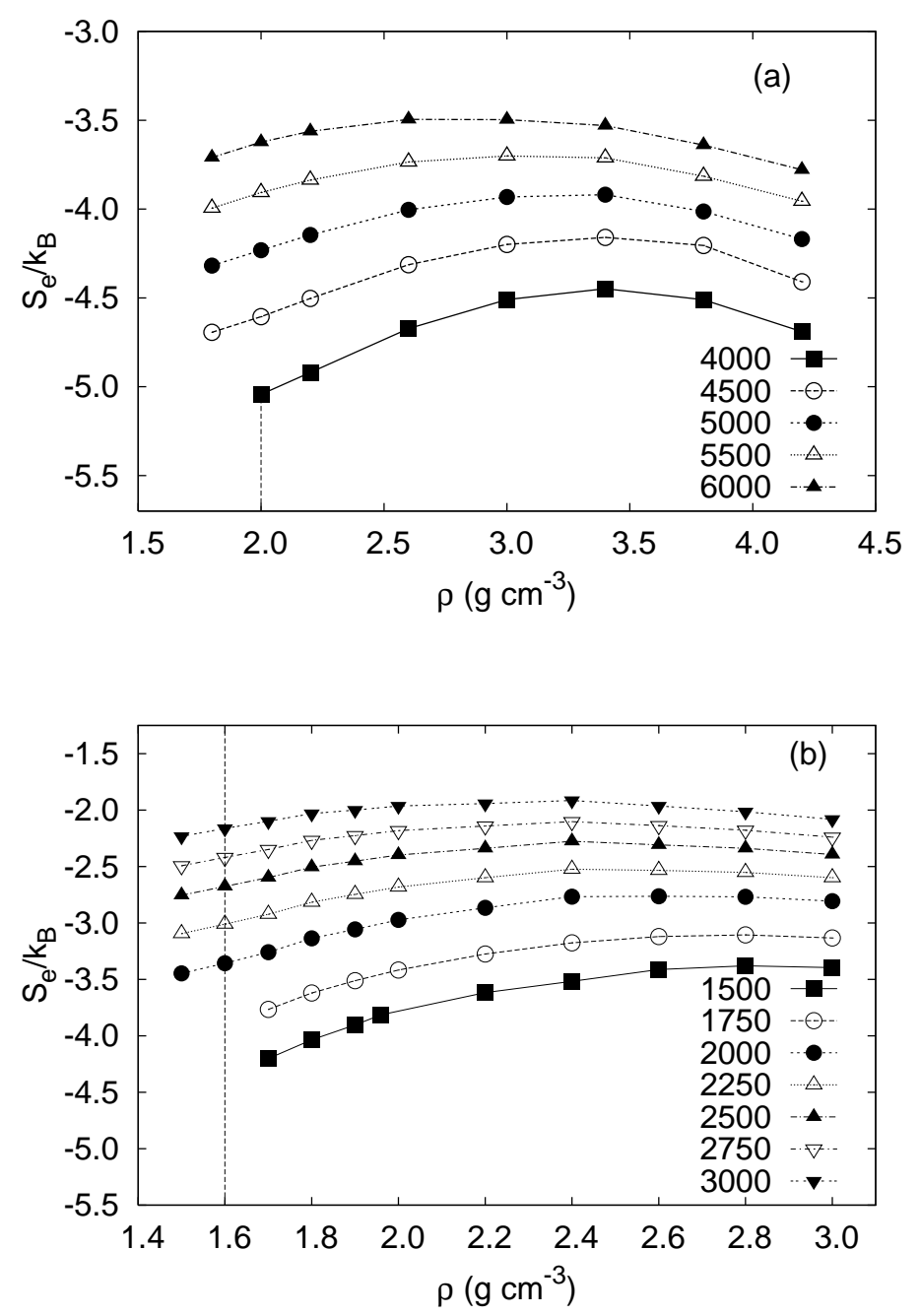
FIG. 2:
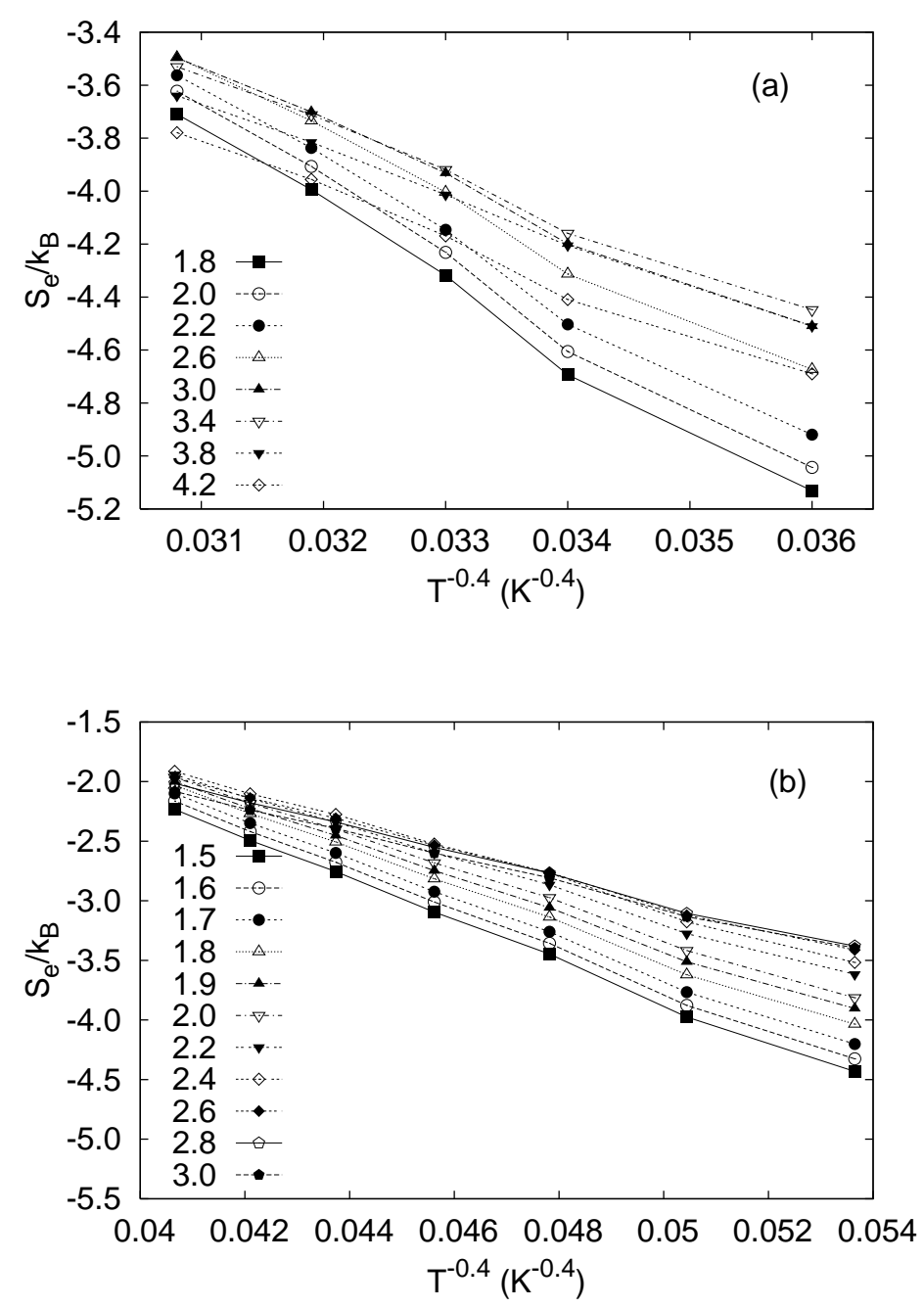
FIG. 3:
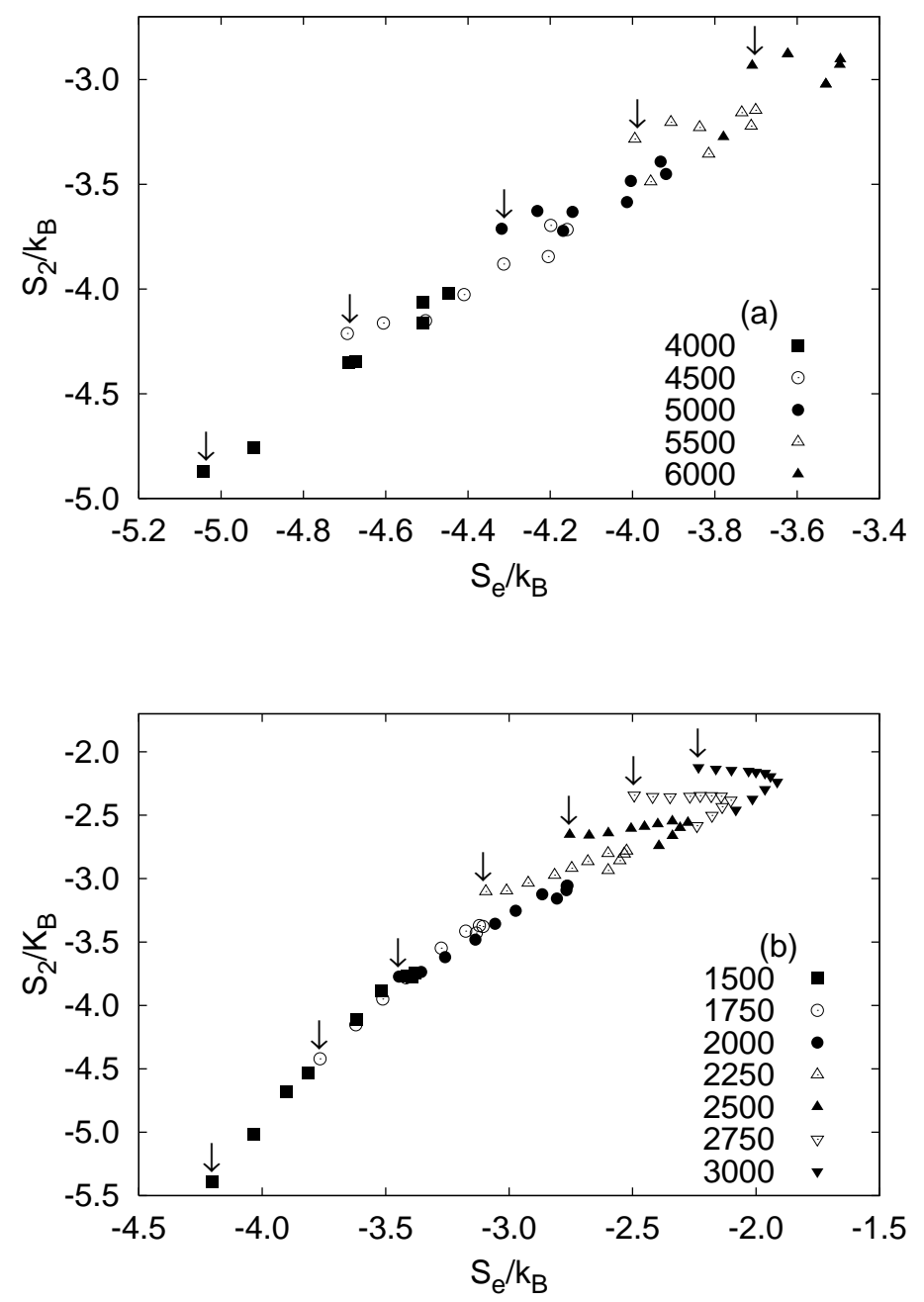
FIG. 4:
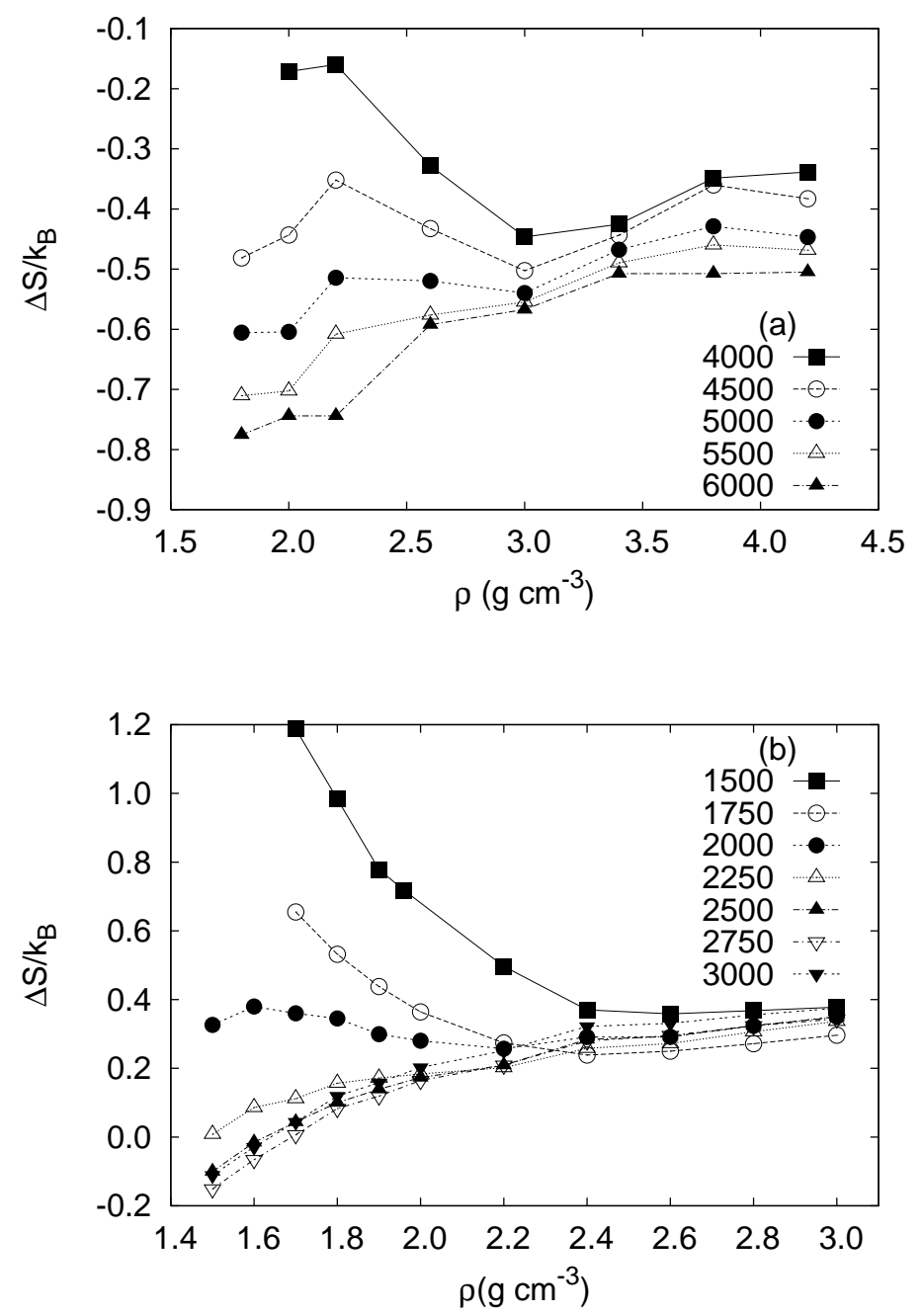
FIG. 5:
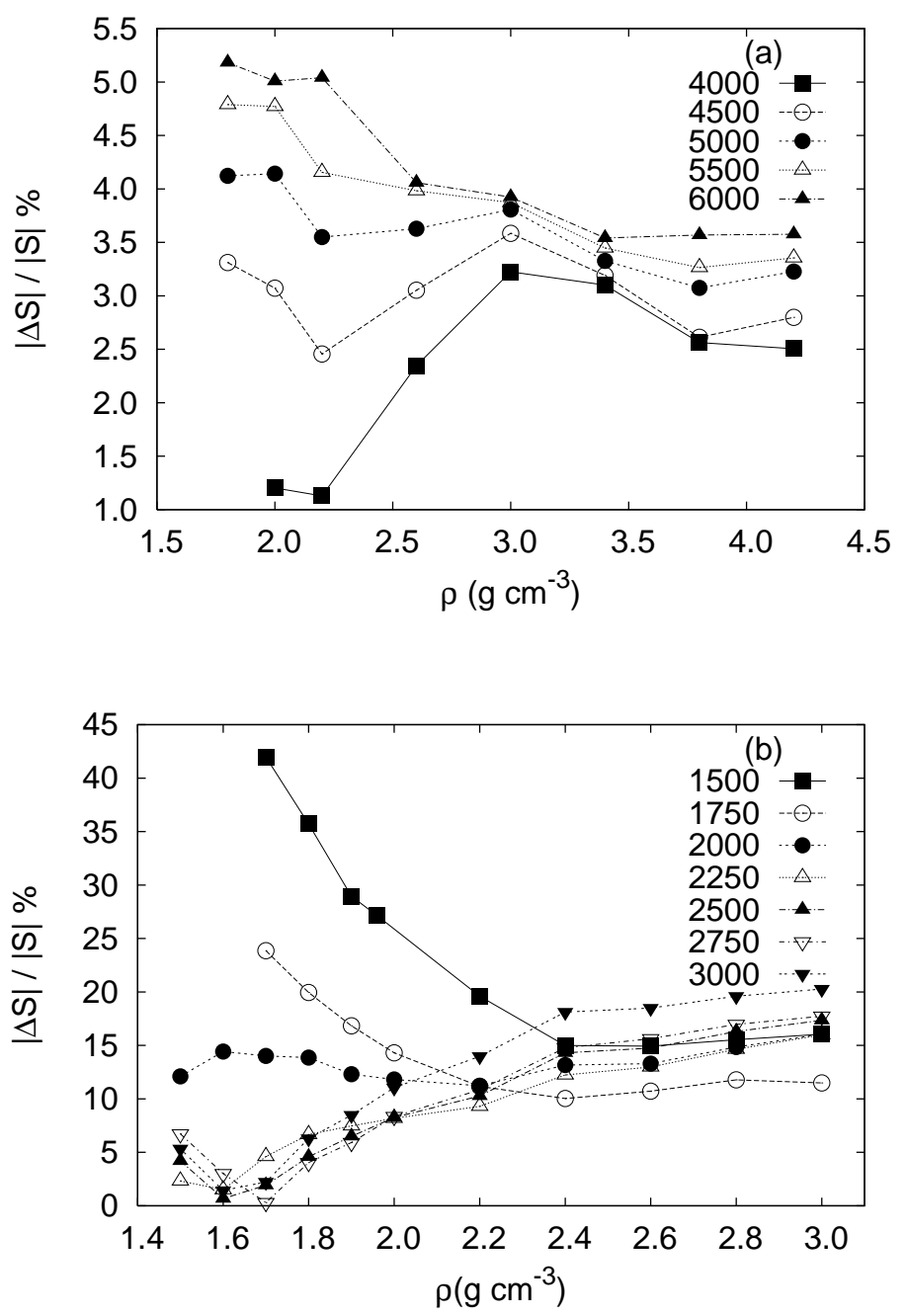
FIG. 6:
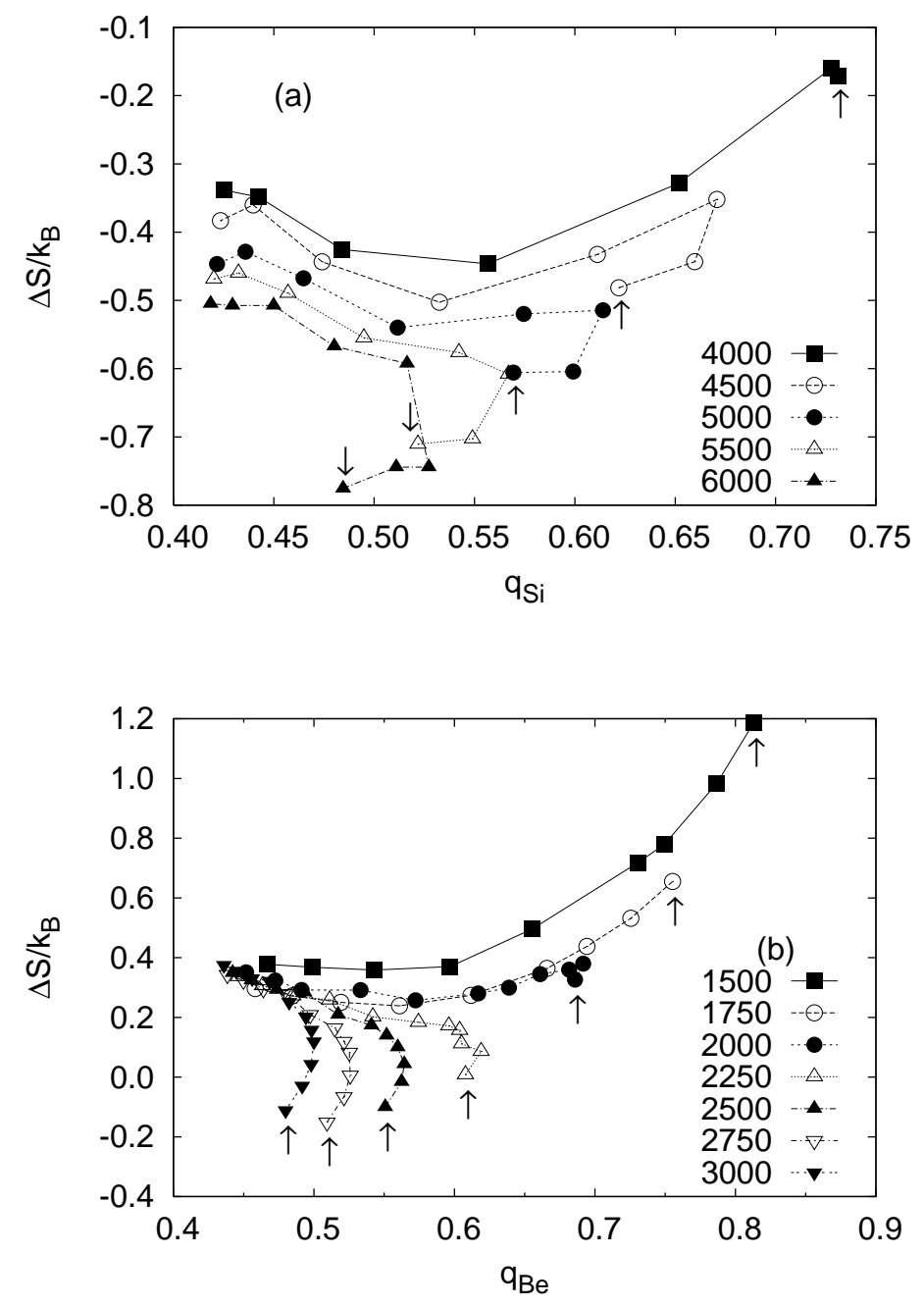
FIG. 7:
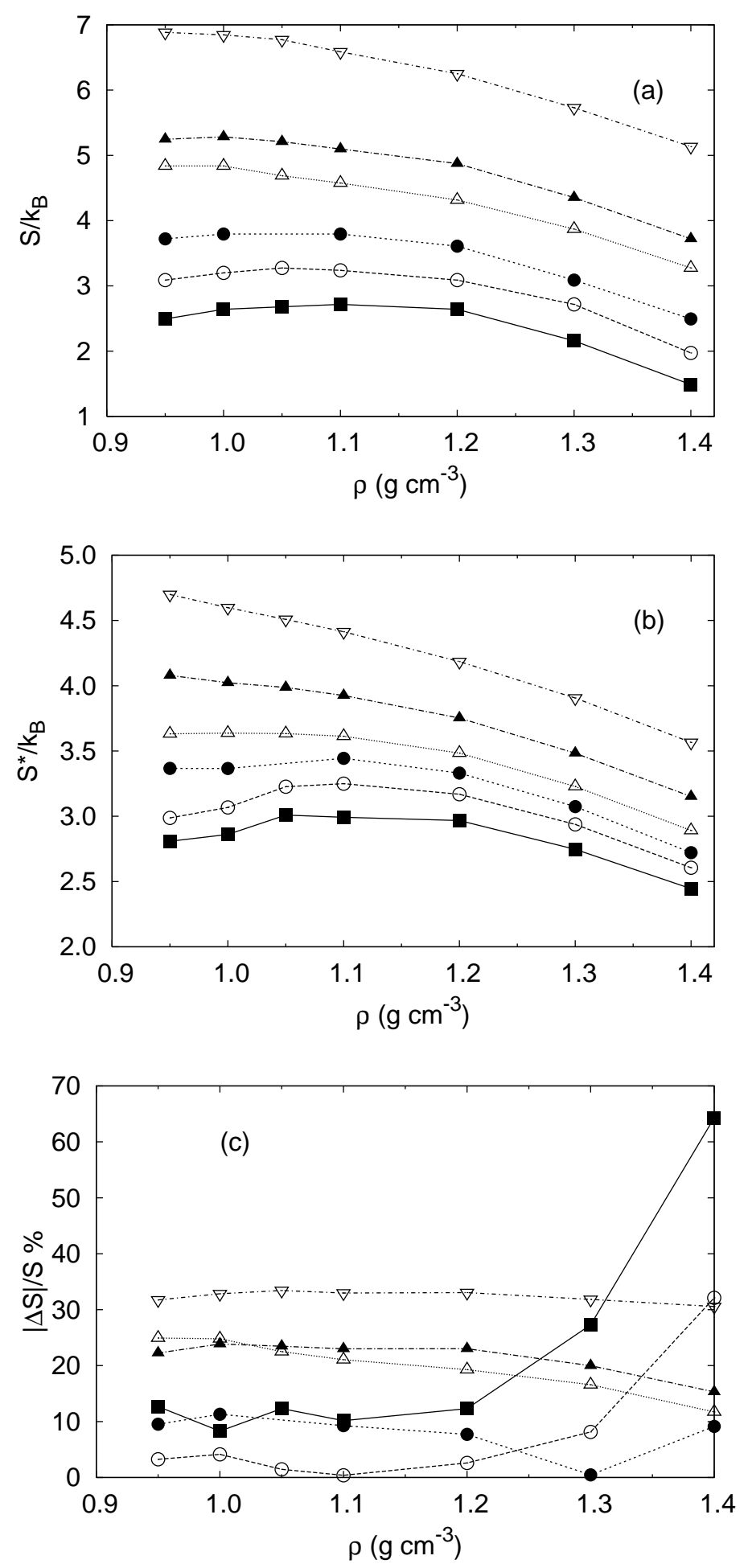
FIG. 8:

Figure 8 (a)

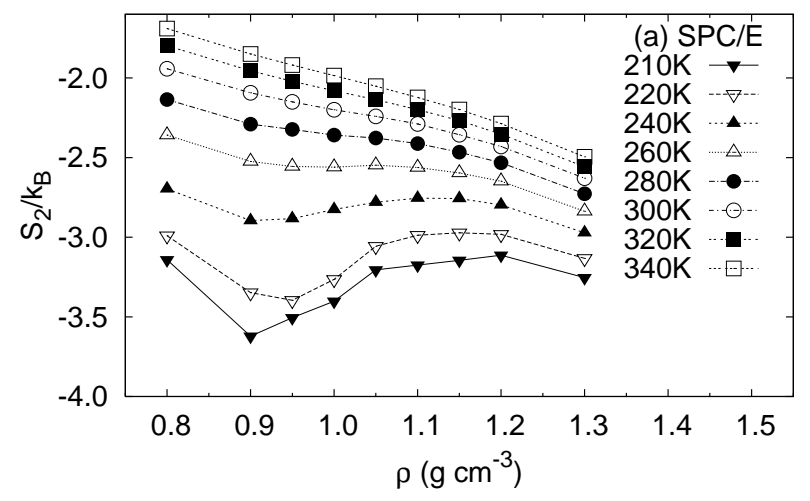

Figure 8 (b)

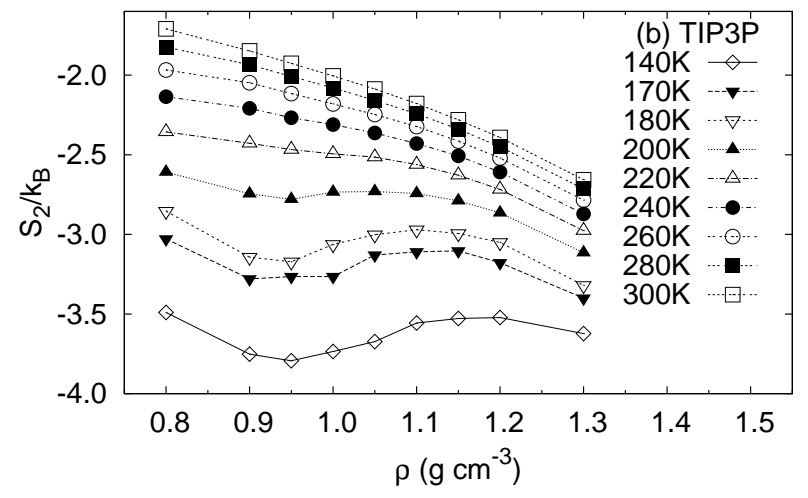

Figure 8 (c)

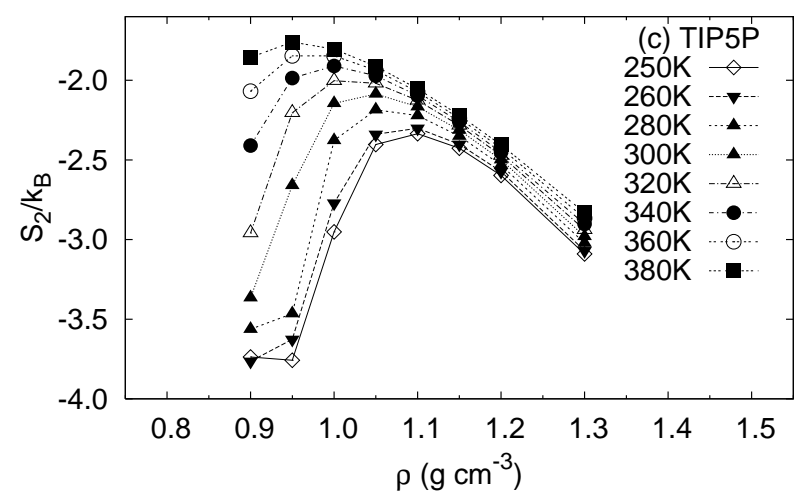

